\title{
Alansmia, a new genus of grammitid ferns (Polypodiaceae) segregated from Terpsichore
}

\author{
Michael Kessler ${ }^{1}$, Ana Laura Moguel Velázquez ${ }^{2,3}$, Michael Sundue ${ }^{4}$, \\ AND Paulo H. LabiaK ${ }^{5}$ \\ ${ }^{1}$ Systematic Botany, University of Zurich, Zollikerstrasse 107, CH-8008, Zurich, Switzerland; \\ e-mail: michael.kessler@systbot.uzh.ch \\ ${ }^{2}$ Department of Systematic Botany, Albrecht-von-Haller-Institute of Plant Sciences, Georg-August- \\ University, Untere Karspüle 2, 37073, Göttingen, Germany \\ ${ }^{3}$ Present Address: Pfefferackerstr. 22, 45894, Gelsenkirchen, Germany; e-mail: moguelve@yahoo.com \\ ${ }^{4}$ The New York Botanical Garden, 200th St. and Southern Blvd., Bronx, NY 10458, USA; \\ e-mail: msundue@nybg.org \\ ${ }^{5}$ Departamento de Botânica, Universidade Federal do Paraná, Caixa Postal 19031, 81531-980, \\ Curitiba, PR, Brazil; e-mail: plabiak@ufpr.br
}

\begin{abstract}
Alansmia, a new genus of grammitid ferns is described and combinations are made for the 26 species known to belong to it. Alansmia is supported by five morphological synapomorphies: setae present on the rhizomes, cells of the rhizome scales turgid, both surfaces of the rhizome scales ciliate, laminae membranaceous, and sporangial capsules setose. Other diagnostic characters include pendent fronds with indeterminate growth, concolorous, orange to castaneous rhizome scales with ciliate or sometimes glandular margins, hydathodes often cretaceous, and setae simple, paired or stellate. The group also exhibits the uncommon characteristic of producing both trilete and apparently monolete spores, sometimes on the same plant. New combinations are made for Alansmia alfaroi, A. bradeana, A. canescens, A. concinna, A. contacta, A. cultrata, A. dependens, A. diaphana, A. elastica, A. glandulifera, A. heteromorpha, A. immixta, A. kirkii, A. lanigera, A. laxa, A. longa, A. monosora, A. reclinata, A. semilunaris, A. senilis, A. smithii, A. spathulata, A. stella var. stella, A. stella var. flava, A. turrialbae, A. variabilis, A. xanthotrichia. Lectotypifications are made for Alansmia concina, A. variabilis, Polypodium ciliare, P. flexile, and P. ovalescens. The genus is named in honor of pteridologist Alan R. Smith.
\end{abstract}

Key Words: Africa, Grammitidaceae, Lellingeria, Leucotrichum, Neotropics, taxonomy.

Grammitid ferns are a clade of about 750 species (Parris, 2009) characterized by having green, tetrahedral spores (Mettenius, 1866), sporangial stalks of one row of cells (Wilson, 1959), and fronds that lack scales but typically bear uniseriate, pluricelluar setae (Ching, 1940; Holttum, 1947). They have been treated as a distinct family (Newman, 1840; Ching, 1940; Parris, 1990a; Ranker et al., 2004) or as a subfamily of the Polypodiaceae (e.g., Tryon \& Tryon, 1982; Lellinger, 1989). Phylogenetic studies using chloroplast DNA sequence data have shown that the grammitids are a monophyletic lineage nested within the Polypodiaceae (Hasebe et al., 1995; Ranker et al., 2004; Schneider et al., 2004). Generic delimitation within grammitid ferns is highly controversial, with only one genus recognized by Tryon and Tryon (1982), four genera by Parris (1990b), 12 by Copeland (1947), 18 by Parris (2003), and 25 by Parris (2009). The difficulty in sorting grammitid ferns into genera is due to homoplasy of obvious 
morphological traits such as blade dissection and the rather obscure nature of taxonomically informative traits such as hydathodes, rhizome symmetry, root insertion, or even presence of fungal bodies on the blades (Smith, 1993; Ranker et al., 2004; Sundue et al., 2010). In a series of papers revising the neotropical grammitids based on more technical characters, Bishop (1988, 1989), Smith et al. (1991), Bishop and Smith (1992), Smith (1992, 1993), and Smith and Moran (1992), recognized ten genera. Three other neotropical genera have been described since then (Murillo \& Smith, 2003; Sundue, 2010a; Labiak et al., 2010).

One of these neotropical genera, Terpsichore, was described by Smith (1993) to accommodate about 50 species characterized by hydathodes that often produce a calcareous deposit, reddish to castaneous (less often hyaline) setae mostly 1-3 $\mathrm{mm}$ long on the leaves, concolorous rhizome scales that are orange to castaneous or blackish and usually setulose along the margins, and pinna segments with free, unbranched, pinnate venation. Smith (1993) recognized five informal species groups within Terpsichore and suggested that these may be regarded as natural sections of the genus. These groups were distinguished by characters such as leaf orientation (pendent to erect), blade growth (determinate or indeterminate), rhizome symmetry, color, and margin, and cell type of the rhizome scales, presence or absence of setae on the sporangia, presence or absence of black fungal fruiting bodies (Acrospermum maxonii Farlow ex Riddle), length of petioles, degree of development of the proximal pinnae, whether the setae of the fronds are solitary or clustered, and presence or absence of calcareous secretions on the hydathodes. Most of these characters were later found to be homoplastic, plesiomorphic, or inconsistently distributed among species. When subject to cladistic analysis, based either on DNA sequence data (Ranker et al., 2004; Sundue et al., 2010) or entirely upon morphological characters (Sundue, 2010b; Sundue et al., 2010), the genus is resolved as polyphyletic. New combinations (Labiak \& Matos, 2007) and a new genus, Ascogrammitis (Sundue, 2010a) have been described in efforts to resolve the polyphyly of Terpsichore, but additional problems remain.

As currently understood, the species of Terpsichore sensu Smith (1993) belong to six separate clades within the grammitid ferns (Ranker et al., 2004; Labiak et al., 2010; Sundue et al., 2010). These clades correspond in part to informal groups recognized in Smith's (1993) subgeneric classification. Two of the groups (groups 2 and 4, plus two species from group 3) form a clade with Melpomene A.R. Sm. \& R.C. Moran and part of Lellingeria A. R. Sm. \& R. C. Moran. Species corresponding to one of these clades have been combined in Ascogrammitis (Sundue, 2010a). Group 5 was found nested within Micropolypodium Hayata, and two of its species have been combined there (Labiak \& Matos, 2007). Of the remaining two species groups of Terpsichore, group 1, which includes the type species of the genus (T. asplenifolia (L.) A.R. Sm.), consists of about 12 species and remains as originally defined by Smith (1993). The last group (group 3 pro parte), the T. lanigera group, was recovered as monophyletic by Ranker et al. (2004) with strong branch support values $(100 \%$ parsimony bootstrap, 1.0 posterior probability, Bremer support of 15). This group differs from Smith's (1993) circumscription by the exclusion of two aberrant species (T. delicatula and T. subscabra) that form the Terpsichore subscabra clade. Monophyly of the T. lanigera group was further tested by Labiak et al. (2010) who included seven species in their molecular phylogenetic study focused on circumscribing its sister clade, Leucotrichum, a new genus of five species which they segregated from Lellingeria. Both the monophyly of the T. lanigera group and its relation as sister to Leucotrichum had high branch support values (parsimony bootstrap $100 \%$, posterior probably 1.0) in analyses using chloroplast markers $(a t p B, r b c L)$ and 87 morphological characters. Two morphological synapomorphies further support the sister relationship of Leucotrichum to the T. lanigera group, these being radially symmetrical rhizomes and setae that are hyaline or whitish (Labiak et al., 2010).

The Terpsichore lanigera group is morphologically distinctive and supported by five 

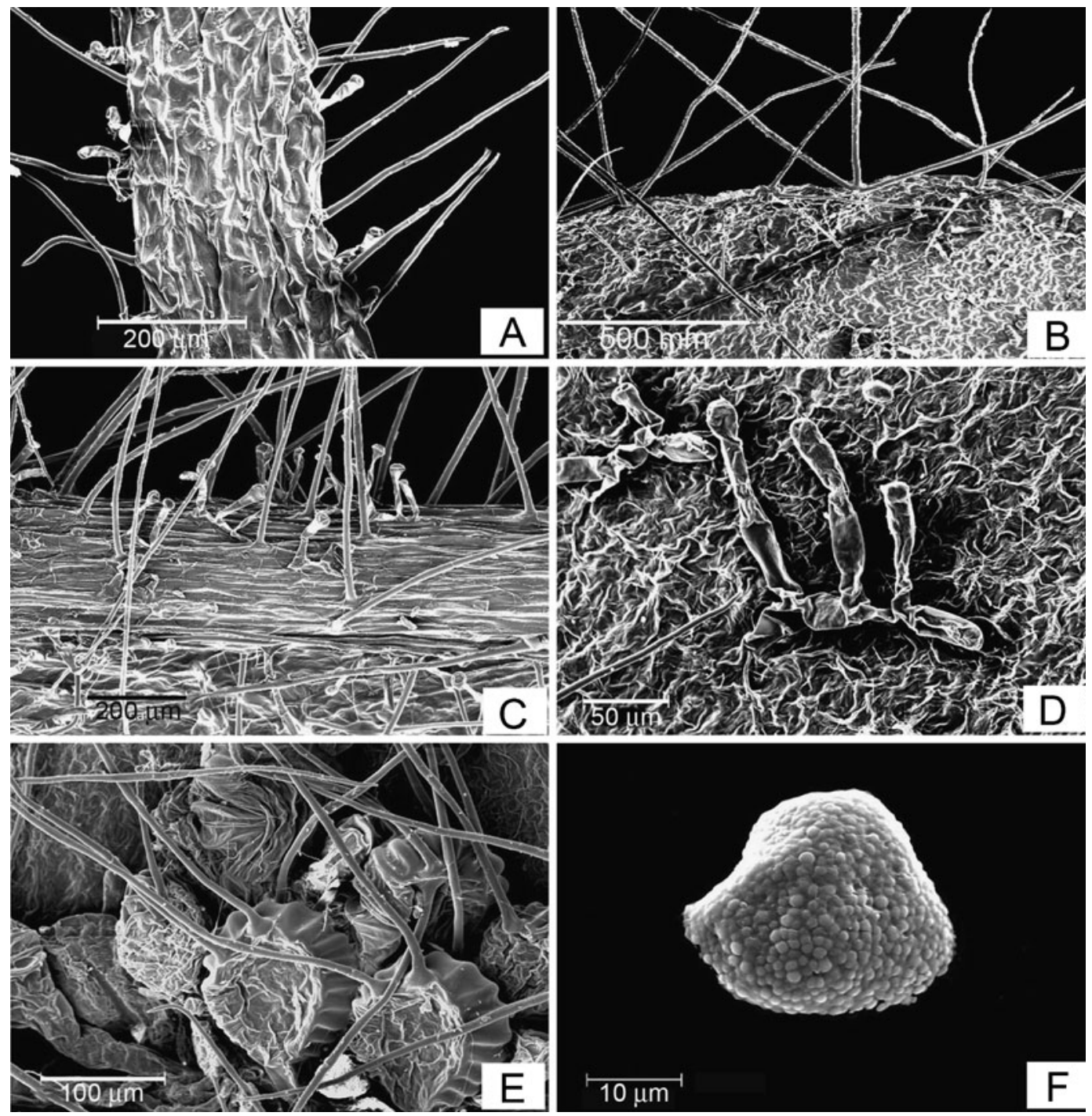

Fig. 1. Characters of Alansmia. A-C. A. reclinata. A. Rhizome scale with cilia and glandular cells in the margin. B. Branched setae in the segment margin. C. Simple setae and branched hairs on the rachis. D-E. A. bradeana. D. Branched hair of the laminar tissue. E. Sporangial capsule with cilia. F. A. reclinata, spore. (A-C from Labiak 964, UPCB; D-E from Prance 10075, NY; F from Labiak 964, UPCB.).

synapomorphies: setae present on the rhizomes, rhizome scales with turgid cells and ciliate adaxial and abaxial surfaces, membranaceous laminae, and episorangial setae (Labiak et al., 2010) (Figs. 1E, 2H, 3J). Several other characters, which occur frequently among the species, are also diagnostic, including pendent fronds with indeterminate growth (Figs. 2B, C, G, 3A, C, K), concolorous (orange to castaneous) rhizome scales with ciliate or sometimes glandular margins
(Figs. 1A, 2A) (scales absent in a few species), hydathodes that are often cretaceous (Fig. 2F), branched hairs on the rachis and laminar tissue (Fig. 1D), and setae that are simple (Fig. 1C), paired, or stellate (Figs. 1B, 2D, 3D, G, H).

In keeping with the recent consensus of dividing the grammitid ferns into genera of tractable size defined by unique combinations of morphological characters (Smith, 1993; Parris, 2003, 2007; Ranker et al., 2004; Sundue, 2010a; Labiak et al., 2010), this 

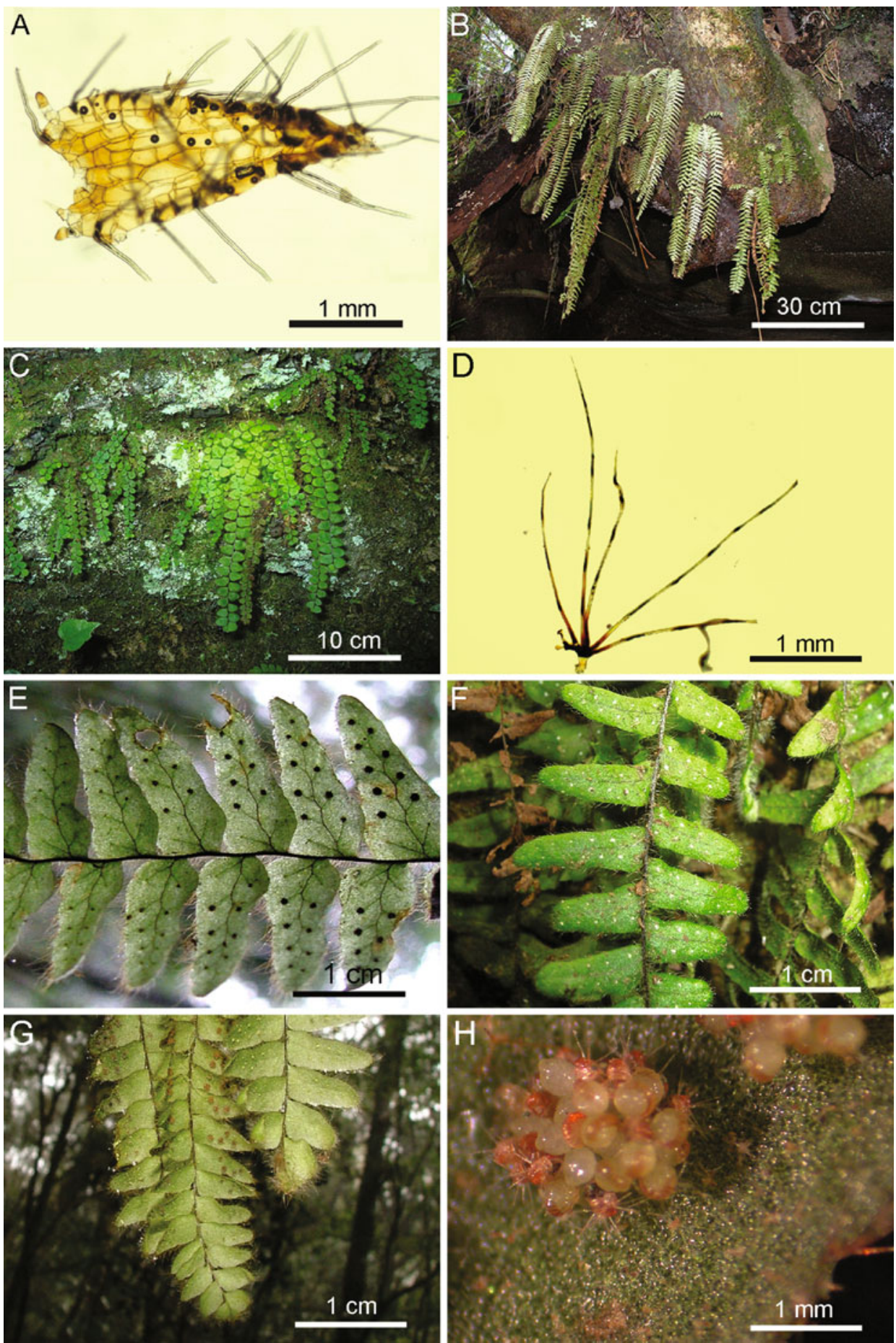

Fig. 2. Characters of Alansmia. A. Alansmia diaphana, rhizome scale. B. A. Reclinata, habit. C. A. senilis. D. A stella var. Stella, rachis seta. E. A. Reclinata, detail of the abaxial side of the lamina. F. A. turrialbae. G. A. reclinata, detail of apex and lower surface. H. A. reclinata, sorus showing the ciliate sporangial capsules. (A from Moguel 32, GOET; C from Sundue 1682, NY; D from van der Werff 6448, MO; F from Sundue 1740, NY; B, E, G, and H without voucher, from Paraná, Brazil). 


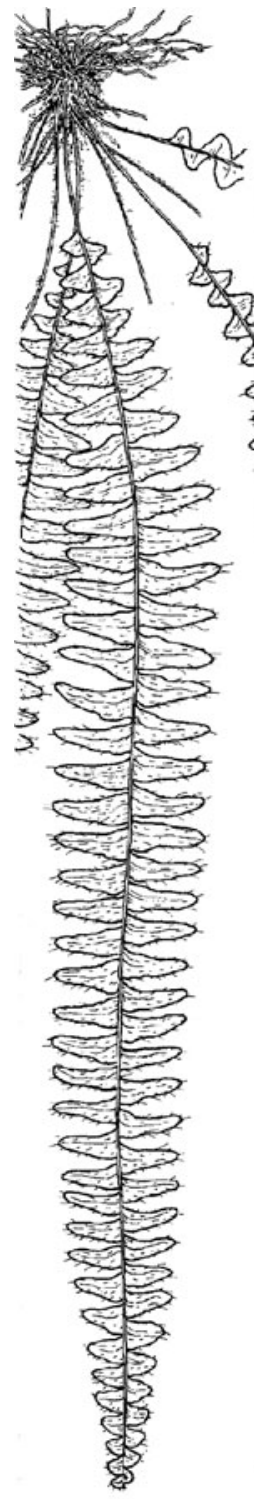

A

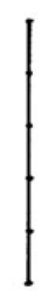

$5 \mathrm{~cm}$.
B

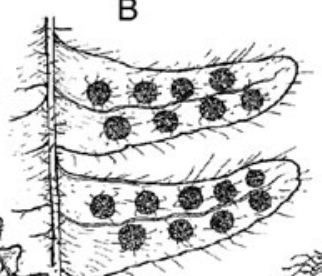

$1 \mathrm{~mm}$

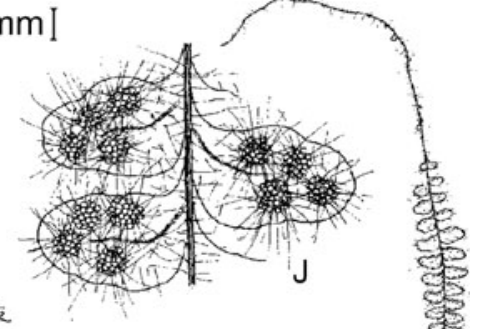

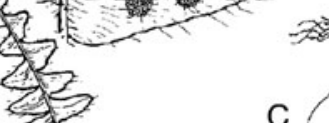

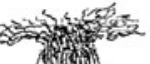

C
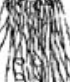

9.<smiles>C1CCC1</smiles>
23 10.
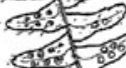
?
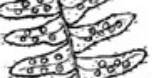

63्य

m:

एक्ष

सू:

एक्षा किष

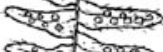

सम

सक 10

कातिक्ष

द्ये

go

पन

सम

कु

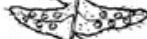

एक

क्ष

एक्ष

इद्धी

एक्षेत्र

सु क्षेक

200
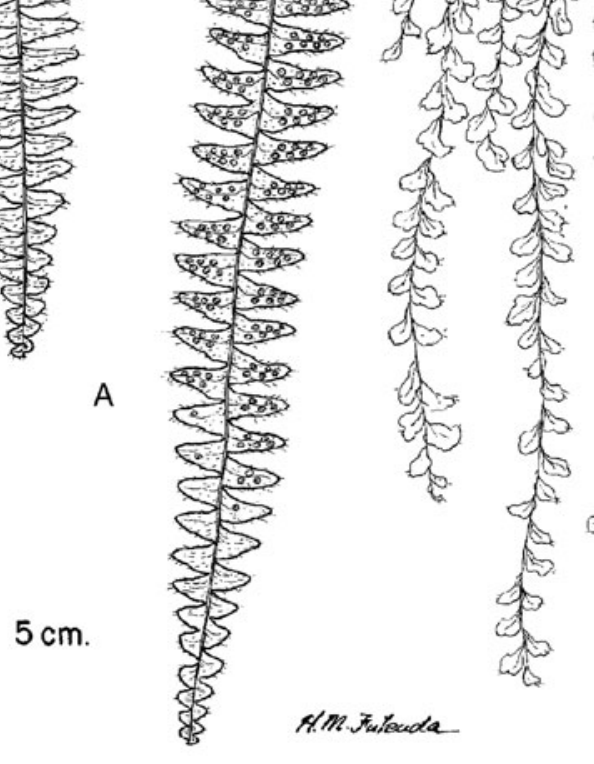

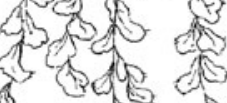
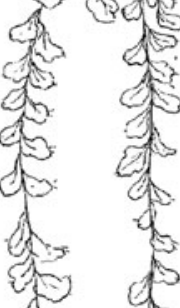

cos

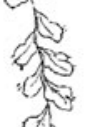

a

秦 G

$\mathrm{K}$

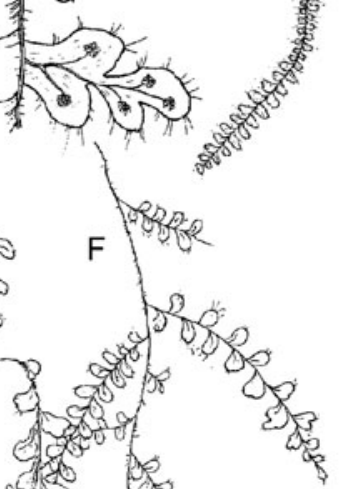

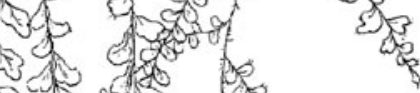

Fig. 3. A-B. Alansmia cultrata. A. Habit. B. Detail of abaxial lamina. C-H. A. spathulata. C. Habit. D, E. Detail of abaxial lamina. F. Frond showing branched rachis. G and H. Segment detail. J-K. A. senilis. J. Detail of abaxial lamina. K. Habit. (A-B from Mickel 6737, NY; C-E from Pringle 4145b, NY; F-H from Bourgeau s.n., NY; J-K from Breedlove 68357, UC.). 
easily diagnosed and well-supported group deserves recognition at the generic level. Therefore, we here describe Alansmia and make the relevant combinations. The description of the new genus is credited to all four authors because each made a significant contribution for this part of the study, whereas the new combinations are credited to only Moguel and Kessler because they conducted the taxonomic revision of the species in this group.

\section{Taxonomic treatment}

Alansmia M. Kessler, Moguel, Sundue \& Labiak, gen. nov. Type: Alansmia lanigera (Desv) Moguel \& M. Kessler. Basionym: Polypodium lanigerum Desv., Ges. Naturf. Freunde Berlin Mag. Neueste Entdeck. Gesamte Naturk. 5: 316. 1811.

Plantae epiphyticae, raro rupestres vel terrestres; rhizomata breve repentia vel decumbentia, radiatim symmetrica, squamata vel setosa, vel in ambobus statibus; squamae rhizomatis luteolae, castaneae vel nigrescentes, non clathratae, pilis margine vel raro in pagina instructae; folia pendentia, indeterminate crescentia, plerumque pinnatisecta pinnis proximalibus gradatim reductis, adaxialiter hydathodis instructa, interdum cum secretionibus calcareis; petioli breves vel brevissimi $(<1 \mathrm{~cm}$ longis); petioli laminae rhachidesque cum pilis simplis vel furcatis, hyalinis vel atropurpureis vestiti; sporangia vulgo setosa; sporae triletae vel ut videtur monoletae.

Plants epiphytic, rarely saxicolous or terrestrial; roots non-proliferous; rhizomes short creeping or very short creeping to suberect, usually radially symmetric, with ventral or radial root insertion, branch buds apparently absent, scales present or sometimes absent, setae present or absent, the scales basifixed, concolorous, dull to shiny, yellowish, orangish, castaneous or very rarely blackish, with variously colored setae (hyaline to atropurpureous) and/or hyaline to orangish hairs on the margins and sometimes surfaces; blades pendent, of indeterminate growth, not articulate to rhizome; petioles with a single vascular bundle, usually short to very short ( $<1 \mathrm{~cm}$ long), setose and puberulent, the setae simple, spreading, $0.5-3.5 \mathrm{~mm}$, hyaline to castaneous, the hairs $0.1-0.2(-0.3) \mathrm{mm}$, branched or unbranched; blades proximally gradually reduced, lacking mycelia and black clavate ascomes of the fungus Acrospermum maxonii, pinnatisect (except in the case of one very deeply pinnatifid species) to 1-pinnate, rarely 1-pinnate-pinnatifid, forking in two species (Fig. 3F), essentially monomorphic, setose and/or puberulent, the setae stramineous, hyaline, yellowish, orangish, castaneous, deep red or atropurpureous, simple, paired, irregularly branched or clustered on pinna surfaces, pinna margins, and rachises, or rarely on the abaxial surfaces of the rachises, also growing out of the transverse walls of catenate axes made of basal cells, up to $1.5 \mathrm{~mm}$ long, the hairs simple or branched, hyaline to orangish or rarely whitish-sericeous, tubular to globose, sometimes eglandular; veins free, pinnate (Fig. 2E); hydathodes present, sometimes cretaceous (producing whitish lime dots); sori round, not sunken into the blade tissue, without paraphyses; sporangia setose or rarely glabrous; spores tetrahedral-globose to ellipsoid or rarely globose, either monolete or trilete; $n=37$ (Evans, 1963).

Etymology.-We dedicate this genus to Alan R. Smith in recognition of his exceptional work on neotropical ferns, particularly the grammitids.

Distribution.-Alansmia comprises 26 species of which 25 occur in the New World tropics and two in Africa, Madagascar, and the Mascarenes. One of the latter species (A. elastica) also occurs in the Neotropics.

Both Alansmia and its sister clade, Leucotrichum, have soft whitish setae, which are uncommon among grammitid ferns, which typically have setae reddish to castaneous and stiff (e.g., Ceradenia, Micropolypodium, Prosaptia). Leucotrichum, however, differs from Alansmia by fronds usually less than $10 \mathrm{~cm}$ long with determinate growth, rhizome scales blackish, strongly clathrate, eciliate on both surfaces, and the hairs 3-celled, 1-furcate, the longer branch cell being acicular.

Whitish setae are also present in the Asian genera Calymmodon and Tomophyllum (Parris, 1990b), and some species of the latter resemble Alansmia. The resemblance is particularly strong in species of Tomophyllum that have branched hairs, and pendant, indeterminate, 
and membranaceous laminae (i.e., Tomophyllum beleense (Copel.) Parris, T. polytrichum (Copel.) Parris). However, the setae of Tomophyllum are simple, unlike those of Alansmia that are branched.

The spores in Alansmia vary from tetrahedral-globose to ellipsoid or reniform in shape and from 25-60 $\mu \mathrm{m}$ long. Bicellular spores occur frequently in Alansmia, particularly in species with ellipsoid spores. Sometimes the usually trilete spores appear monolete or nearly so (Wagner, 1985). These are not truly monolete, but have an inconspicuous third "arm" of the laesura which departs at a low angle to the other two arms, making a narrow Y-shape instead of the typical trilete mark which has roughly equal $120^{\circ}$ angles. This phenomenon can be observed in ellipsoid or reniform spores, whereas in tetrahedral-globose spores a triradiate laesura is typical. Similar variation in spore laesurae was reported to occur in Dasygrammitis brevivenosa (Alderw.) Parris by Nayar \& Devi (1965) (as Ctenopteris brevivenosa (Alderw.) Holttum), suggesting that it may not be unique to Alansmia; however, Wagner (1985) was unable to confirm this observation.

\section{NEW COMBINATIONS AND SYNONYMY}

Alansmia alfaroi (Donn. Sm.) Moguel \& M. Kessler, comb. nov. Polypodium alfaroi Donn. Sm., Bot. Gaz. 33: 262. 1902, as "alfari". Ctenopteris alfaroi (Donn. Sm.) Copel., Philipp. J. Sci. 84: 433. 1956. Grammitis alfaroi (Donn. Sm.) C. V. Morton, Contr. U.S. Natl. Herb. 38: 103. 1967. Terpsichore alfaroi (Donn. Sm.) A. R. Sm., Novon 3: 485. 1993. Type: Costa Rica. Cártago: Sierra Alta de Navarro, $1400 \mathrm{~m}$ [as indicated on sheet, protologue erroneously gives $2000 \mathrm{~m}$ ], May 1901, $A$. Alfaro 8063 (holotype: US).

Polypodium oligosorum Mett. ex Kuhn, Linnaea 36: 132. 1869, nom. illeg., non Klotzsch, 1847.

Alansmia bradeana (Labiak) Moguel \& $\mathrm{M}$. Kessler, comb. nov. Terpsichore bradeana Labiak, Brittonia 52: 251. 2000. Type: Brazil. Roraima: Serra dos Surucucus, $02^{\circ} 42-47^{\prime} \mathrm{N}, 63^{\circ} 33-36^{\prime} \mathrm{W}, 19$ Feb
1969, G. T. Prance, J. R. Steward, J. F. Ramos \& L. G. Farias 10075 (holotype: INPA; isotypes: $\mathrm{K}, \mathrm{NY}$ ).

Alansmia canescens (A. Rojas) Moguel \& M. Kessler, comb. nov. Terpsichore canescens A. Rojas, Métodos en Ecología y Sistemática 3: 14. 2008. Type. Colombia. Santander: Municipio de Charalá, Inspección Virolín, Vereda El Volcán, 1900 m, 30 Jun 1983, J. Torres 2575 (holotype: COL-n.v).

Alansmia concinna (A. R. Sm.) Moguel \& M. Kessler, comb. nov. Grammitis concinna A. R. Sm., Ann. Missouri Bot. Gard. 76: 338. 1989, nom. nov. for Polypodium concinnum Mett. ex Kuhn, Linnaea 36: 132. 1869, non Willd. 1810. Terpsichore concinna (A. R. Sm.) A. R. Sm., Novon 3: 486. 1993. Type: Venezuela. Aragua: High mountains east of Colonia Tovar, 25 Jun 1854, A. Fendler 212 (lectotype, here designated: $\mathrm{MO}$; duplicates of lectotype: K, GH-n.v., YU-n.v.).

Alansmia contacta (Copel.) Moguel \& M. Kessler, comb. nov. Ctenopteris contacta Copel., Philipp. J. Sci. 84: 447. 1956. Type: Bolivia. La Paz: Prov. Nor Yungas, Yungas, 1890, M. Bang 483 (lectotype, designated by Tryon \& Stolze, 1993: US; duplicates of lectotype: B, F-n.v., K, N).

Alansmia cultrata (Bory ex Willd.) Moguel \& M. Kessler, comb. nov. Polypodium cultratum Bory ex Willd., Sp. Pl. ed. 4. 5: 187. 1810. Xiphopteris cultrata (Bory ex Willd.) Schelpe, Bol. Soc. Brot., ser. 2, 41: 217. 1867. Ctenopteris cultrata (Bory ex Willd.) Copel., Gen. Fil. 219. 1947. Grammitis cultrata (Bory ex Willd.) Proctor, Rhodora 63: 35. 1961. Terpsichore cultrata (Bory ex Willd.) A. R. Sm., Novon 3: 486. 1993. Type: Jamaica or Martinique, without precise locality, $O$. Swartz s.n. (lectotype, designated by Proctor, 1985: B; duplicate of lectotype: S-n.v).

Alansmia dependens (Baker) Moguel \& $\mathrm{M}$. Kessler, comb. nov. Polypodium dependens Baker, Syn. Fil. 335. 1867. Grammitis dependens (Baker) C. V. Morton, Contr. 
U. S. Natl. Herb. 38: 104. 1967. Xiphopteris heteromorpha var. dependens (Baker) Crabbe, Brit. Fern Gaz. 9: 318. 1967. Terpsichore dependens (Baker) A. R. Sm., Novon 3: 486. 1993. Type. Ecuador. Pichincha: Mt. Pichincha, $R$. Spruce 5637 (holotype: K).

Alansmia diaphana (Moguel \& M. Kessler) Moguel \& M. Kessler, comb. nov. Terpsichore diaphana Moguel \& M. Kessler, Phytotaxa 2: 38. 2009. Type. Bolivia. La Paz: Prov. Nor Yungas, hill above Cotapata on La Paz-Caranavi road, $16^{\circ}$ 17'S, 6750'W, $3200 \mathrm{~m}, 15$ Aug 1990, A. Fay \& L. Fay 3031 (holotype: LPB; isotypes: LPB, MO, NY, UC).

Alansmia elastica (Bory ex Willd.) Moguel \& M. Kessler, comb. nov. Polypodium elasticum Bory ex Willd., Sp. Pl., ed. 4. 5: 183. 1810. Polypodium leveilleanum Desv., Mém. Soc. Linn. Paris 6: 232. 1827, nom superfl. Ctenopteris elastica (Bory ex Willd.) Copel., Philipp. J. Sci. 84: 426. 1956. Xiphopteris elastica (Bory ex. Willd.) Alston, Bol. Soc. Brot., ser. 2, 26: 27. 1956. Terpsichore elastica (Bory ex Willd.) A. R. Sm., Novon 5: 21. 1995. Type: Réunion [“Bourbon"]: forêts élevée, J. B. G. M. Bory s.n. (holotype: B-W 19675).

Polypodium mollissimum Fée, Hist. Foug. Antill. [Mém. Foug. 11]: 47, t. 12, f. 2. 1866. Ctenopteris mollissima (Fée) Copel., Philipp. J. Sci. 84: 423. 1956. Grammitis mollissima (Fée) Proctor, Rhodora 63: 35. 1961. Terpsichore mollissima (Fée) A. R. Sm., Novon 3: 487. 1993. Type: Guadeloupe: Bois élevés de la [illegible] St. Louis, bras [illegible], 1861, F. L'Herminier 13 (lectotype, designated by Proctor, 1985: P; duplicates of lectotype: B, K-n.v., US-n.v.).

Polypodium flexile Fée, Synopsis Filicum 54 [Mem. Foug. 2]: 9, t. 2, f. 3. 1854. Type: Mauritius: $F . W$. Sieber 54 (lectotype, here designated: B).

Terpsichore cocosensis A. Rojas, Brenesia 45-46: 47. 1996. Type: Costa Rica. Puntarenas: Isla del Coco, Cerro Pelón, 5`32'40”N, 8703’20”W, 200-400 m, 13 Jun 1994, E. Lépiz 337 (holotype: INB-n.v.; isotypes: CR-n.v., MO-n.v., UC).

Terpsichore esquiveliana A. Rojas, Rev. Biol. Trop. 49: 451. 2001. Type: Costa Rica. Cartago: Paraíso, Parque Nacional Tapantí, Sendero los Palmitos

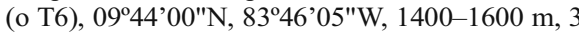
Sep 1997, A. Rojas 3785 (holotype: INB; isotypes: CR-n.v., MO-n.v., UC-n.v.).
Terpsichore lobulata A. Rojas, Métodos en Ecología y Sistemática 3: 14. 2008. Type. Colombia. Norte de Santander: Cordillera Oriental, región del Sararé, Hoya del Río Cubugón, El Indio, 420480 m, 13-17 Nov 1941, J. Cuatrecasas 13089 (holotype: COL-n.v.).

Alansmia glandulifera (A. Rojas) Moguel \& M. Kessler, comb. nov. Terpsichore glandulifera A. Rojas, Lankesteriana 6: 96. 2006. Type: Costa Rica. Cartago: Paraíso, Parque Nacional Tapantí, cuenca del Río Reventazón, Orosi, sendero Palmitos o T6, 9०43'35"N, $83^{\circ}$ 46'28"W, 1460-1600 m, 3 Sep 1997, A. Rojas \& R. Delgado 3788 (holotype: CR-n.v.; isotypes: INB-n.v., MO-n.v.).

Alansmia heteromorpha (Hook. \& Grev.) Moguel \& M. Kessler, comb. nov. Polypodium heteromorphum Hook. \& Grev., Icon. Fil. 1: t. 108. 1829. Ctenopteris heteromorpha (Hook. \& Grev.) Copel., Philipp. J. Sci. 84: 412. 1956. Grammitis heteromorpha (Hook. \& Grev.) C. V. Morton, Contr. U. S. Natl. Herb. 38: 102. 1967. Xiphopteris heteromorpha (Hook. \& Grev.) Crabbe, Brit. Fern Gaz. 9: 318. 1967. Terpsichore heteromorpha (Hook. \& Grev.) A. R. Sm., Novon 3: 486. 1993. Type: Ecuador. Pichincha: Mt. Pichincha, between Mt. Chimborazo and Pichincha, $W$. Jameson s.n. (lectotype, designated by Morton, 1967: K; duplicates of lectotype: FI-n.v., NY).

Alansmia immixta (Stolze) Moguel \& M. Kessler, comb. nov. Grammitis immixta Stolze, Fieldiana, Bot., n.s. 32: 115. 1993. Terpsichore immixta (Stolze) A. R. Sm., Novon 3: 487. 1993. Type: Peru. Cuzco: La Convención, Vilcabamba, Ruina Idma-huasi, 4000 m, Jul 1934, G. Bües 2103 (holotype: US; isotypes: F-n.v., GH-n.v., MO, NY).

Alansmia kirkii (Parris) Moguel \& M. Kessler, comb. nov. Terpsichore kirkii Parris, Kew Bulletin 57: 431. 2002. Type: Malawi. Shire, Zambesi Exp., J. Kirk s.n. (holotype: K).

Alansmia lanigera (Desv.) Moguel \& M. Kessler, comb. nov. Polypodium lanigerum Desv., Ges. Naturf. Freunde Berlin 
Mag. Neueste Entdeck. Gesamte Naturk. 5: 316. 1811. Ctenopteris lanigera (Desv.) Copel., Philipp. J. Sci. 84: 420. 1956. Grammitis lanigera (Desv.) C. V. Morton, Contr. U.S. Natl. Herb. 38: 105. 1967. Xiphopteris lanigera (Desv.) Crabbe, Brit. Fern Gaz. 9: 319. 1967. Terpsichore lanigera (Desv.) A. R. Sm., Novon 3: 487. 1993. Type: Peru. without precise locality, J. Dombey s.n. [Herbarium Jussieu 1099] (lectotype, designated by Morton, 1967: P; duplicates of lectotype: B, P [P00279983]).

Polypodium sericeolanatum Hooker, Sp. Fil. 4: 221. 1864. Type: Ecuador. Pichincha: ravines of Pichincha, 3900-4000 m, W. Jameson 235 (lectotype, designated by Morton, 1967: K; duplicate of lectotype: B).

Alansmia laxa (C. Presl) Moguel \& M. Kessler, comb. nov. Polypodium laxum C. Presl, Reliq. Haenk. 1: 23, t. 4, f. 1. 1825. Grammitis laxa (C. Presl) C. V. Morton, Contr. U.S. Natl. Herb. 38: 105. 1967. Terpsichore laxa (C. Presl) A. R. Sm., Novon 3: 487. 1993. Type. Peru. without precise locality, T. Haenke s. $n$. (lectotype, designated by Tryon \& Stolze, 1993: PRC; duplicate of lectotype: PR).

Alansmia longa (C. Chr.) Moguel \& M. Kessler, comb. nov. Polypodium longum C. Chr., Index. Fil. 541. 1906, nom. nov. for P. alternifolium Hook. non Willd., 1810. Ctenopteris longa (C. Chr.) Copel., Philipp. J. Sci. 84: 453. 1956. Xiphopteris longa (C. Chr.) Alston, Bull. Jard. Bot. Etat 27: 35. 1937. Terpsichore longa (C. Chr.) A. R. Sm., Novon 3: 487. 1993. Type: Ecuador. Near Esmeraldas and between Cuenca and Guayaquil, 1000-3300 m, W. Jameson s. $n$. (lectotype, designated by Morton, 1967: K, duplicate of lectotype: US-n.v.).

Terpsichore acrosora A. Rojas, Métodos en Ecología y Sistemática 3: 13. 2008. Type: Costa Rica. Limón: Talamanca, Bratsi, Amubri, Alto Lari, Kivut, quebrada innominada, margen derecha del

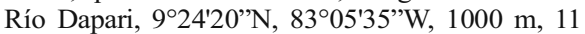
Mar 1992, G. Herrera 5302 (holotype: CR-n.v.; isotypes: INB-n.v., MO).

Alansmia monosora (Moguel \& M. Kessler) Moguel \& M. Kessler, comb. nov. Terp- sichore monosora Moguel \& M. Kessler, Phytotaxa 2: 40. 2009. Type. Ecuador. Pichincha: Andes of Quito, $4000 \mathrm{~m}$, Jun 1877, W. Jameson s.n. [Herbarium Hookerianum 1867] (holotype: K barcode: K000590671; isotype: $\mathrm{K}$ barcode: K000590672 [Herbarium Hookerianum 1854]).

Alansmia reclinata (Brack.) Moguel \& $\mathrm{M}$. Kessler, comb. nov. Polypodium reclinatum Brack., U. S. Expl. Exped. 16: 11. 1854. Ctenopteris reclinata (Brack.) Copel., Philipp. J. Sci. 84: 424. 1956. Terpsichore reclinata (Brack.) Labiak, Brittonia 52: 253. 2000. Type: Brazil. Rio de Janeiro. Serra dos Orgaos, Wilkes Expedition 161. (lectotype, designated by Copeland, 1956: US; duplicates of lectotype: K, NY).

Polypodium ciliare Fée, Crypt. Vasc. Brésil 1: 94, t. 27, f. 2. 1869. Ctenopteris ciliare (Fée) Copel., Philipp. J. Sci. 84: 425. 1955[1956]. Type: Brazil. Rio de Janeiro: Habitat in Brasilia Fluminensi, $A$. Glaziou 961 (lectotype, here designated: P; duplicates of lectotype: BR, NY).

Polypodium ovalescens Fée, Crypt. Vasc. Brésil 1: 94, t. 27, f. 3. 1869. Type: Brazil. Rio de Janeiro: Habitat in Brasilia Fluminensi, A. Glaziou 1722 (lectotype, here designated: $\mathrm{P}$ [P00279989]; duplicates of lectotype: BR, P-n.v. [P00632688]).

Alansmia semilunaris (Moguel \& $\mathrm{M}$. Kessler) Moguel \& M. Kessler, comb. nov. Terpsichore semilunaris Moguel \& M. Kessler, Phytotaxa 2: 42. 2009. Type. Ecuador. Napo: ca. $3 \mathrm{~km} \mathrm{~W}$ of Oyacachi, 00¹2'S, 7806'W, 3550 m, 27 Mar 1996, B. Ståhl \& H. Navarrete 2295 (holotype: AAU; isotype: QCA-n.v.).

Alansmia senilis (Fée) Moguel \& M. Kessler, comb. nov. Polypodium senile Fée, Mém. Soc. Sci. Hist. Nat. Strasbourg 5 [Mém. Foug. 7]: 60, t. 25, f. 1. 1857. Ctenopteris senilis (Fée) Copel., Philipp. J. Sci. 84: 398. 1956. Grammitis senilis (Fée) C. V. Morton, Contr. U.S. Natl. Herb. 38: 103. 1967. Terpsichore senilis (Fée) A. R. Sm., Novon 3: 488. 1993. Type: Colombia. Norte de Santander: Ocaña, L. J. Schlim 364 (holotype: RB).

Polypodium subflabelliforme Rosenst. Repert. Spec. Nov. Regni. Veg. 7: 306. 1909. Ctenopteris 
subflabelliformis (Rosenst.) Copel., Philipp. J. Sci. 84: 400. 1956. Type: Ecuador. Tungurahua: Cerro de Abitagua, Andium Quitensium, $R$. Spruce 5271 (lectotype, designated by Tryon \& Stolze, 1993: K; duplicate of lectotype: NY).

Polypodium subflabelliforme Rosenst. var. minor Rosenst., Spec. Nov. Regni. Veg. 7: 307. 1909. Type: Ecuador: Tungurahua: In Monte Tungurahua, Andium Quitensium, R. Spruce 5272 (holotype: K).

Polypodium senile Fée var. minor Rosenst., Mededeelingen van's Rijks Herbarium 19: 19. 1913. Type: Bolivia. Cochabamba: Yungas de San Mateo, 2700 m, Apr 1911, T. Herzog 1985 (lectotype, designated by Tryon \& Stolze, 1993: L-n.v.; duplicates of lectotype: B, US).

Alansmia smithii (A. Rojas) Moguel \& M. Kessler, comb. nov. Terpsichore smithii A. Rojas, Métodos en Ecología y Sistemática 3: 15. 2008. Type. Costa Rica. Cartago: El Guarco, Madre Selva, km 64, Finca Los Lagos, sendero a orillas del río, 9०40'34'N, 83 52'38' 'W, 2600 m, 21 Apr 1999, A. Rojas \& L. Pacheco 5090 (holotype: CR-n.v.; isotypes: INB-n.v., MO-n.v.).

Alansmia spathulata (A. R. Sm.) Moguel \& M. Kessler, comb. nov. Terpsichore spathulata A. R. Sm., Novon 3: 483-485. 1993. Type: México. State of México: Summit of Sierra de las Cruces, $3800 \mathrm{~m}$, 11 Sep 1892, C. G. Pringle 4145b (holotype: UC; isotypes: $\mathrm{K}, \mathrm{MO}, \mathrm{NY}$, US).

Alansmia stella (Copel.) Moguel \& M. Kessler var. stella, comb. nov. Ctenopteris stella Copel., Philipp. J. Sci. 84: 452. 1956. Terpsichore stella (Copel.) Moguel \& M. Kessler, Phytotaxa 2: 36. 2009. Type: Peru. Cuzco: Valley of Río Urubamba, A. Bües 32 (holotype: US).

Ctenopteris fabispora Copel., Philipp. J. Sci. 84: 457. 1956. Terpsichore fabispora (Copel.) A. Rojas. Type. Panama. Chiriquí: between Alto de Las Palmas and the top of Cerro Horqueta, 2100 2268 m, Mar 1911, W. Maxon 5479 (holotype: US).

Alansmia stella (Copel.) Moguel \& M. Kessler var. flava (Moguel \& M. Kessler) Moguel \& M. Kessler, comb. nov. Terpsichore stella (Copel.) Moguel \& M. Kessler var. flava Moguel \& Kessler,
Phytotaxa 2: 37. 2009. Type: Peru. Amazonas: Road Chachapoyas-Mendoza, a little past Molinopampa, $06^{\circ} 14^{\prime} \mathrm{S}, 77^{\circ}$ 35'W, 2400 m, 15 Mar 1998, H. van der Werff, B. Gray, R. Vasquez \& R. Rojas 15009 (holotype: MO-n.v.; isotype: UC).

Alansmia turrialbae (H. Christ) Moguel \& M. Kessler, comb. nov. Polypodium turrialbae H. Christ, Bull. Soc. Roy. Bot. Belgique 35: Mém 226. 1896. Ctenopteris turrialbae (H. Christ) Copel., Philipp. J. Sci. 84: 419. 1956. Grammitis turrialbae (H. Christ) F. Seym., Phytologia 31: 181. 1975. Terpsichore turrialbae (H. Christ) A. R. Sm., Novon 3: 488. 1993. Type: Costa Rica. Cartago: Troncs d'arbres au pied du volcan de Turrialba, 2750 m, 26 Jan 1889, H. F. Pittier 847 (lectotype, designated by Lellinger, 1989: BR; duplicate of lectotype: US).
Polypodium exsudans H. Christ, Bull. Herb. Boiss. sér. 2, 4: 1103. 1904. Type: Costa Rica. C. Wercklé s.n. (holotype: P).

Alansmia variabilis (Mett. ex Kuhn) Moguel \& M. Kessler, comb. nov. Polypodium variabile Mett. ex Kuhn, Linnaea 36: 133. 1869. Grammitis variabilis (Mett. ex Kuhn) C. V. Morton, Contr. U.S. Natl. Herb. 38: 102. 1967. Terpsichore variabilis (Mett. ex Kuhn) A. R. Sm., Novon 3: 488. 1993. Type: Colombia. [Depto. Caldas or Tolima]: Páramo del Ruiz, Jun 1826, A. W. Purdie s.n. (lectotype, here designated: $\mathrm{K}$; duplicate of lectotype: B).

Alansmia xanthotrichia (Klotzsch) Moguel \& M. Kessler, comb. nov. Polypodium xanthotrichium Klotzsch, Linnaea 20: 376. 1847. Grammitis xanthotrichia (Klotzsch) Duek \& Lellinger, Amer. Fern. J. 68: 120. 1978. Terpsichore xanthotrichia (Klotzsch) A. R. Sm., Novon 3: 488. 1993. Type: Venezuela. Mérida: J. W. K. Moritz 250 (holotype: B).

Polypodium ellipticosorum Fée, Mem. Foug. [Gen. Filic.] 5: 239. 1850. Type: Venezuela. Mérida: 2000 m, N. Funck \& L. J. Schlim 960 (lectotype, here designated: $\mathrm{K}$; duplicate of lectotype: $\mathrm{P}$ ). 


\section{Acknowledgments}

We thank Alan R. Smith for his unconditional support of our pteridological studies. Kessler's research was supported by the German Research Association (DFG), Labiak's research by a grant from the Brazilian CNPq (PED 201782/2008-1), and Sundue's research by a grant from the United States National Science Foundation (NSF) (in the name of Dr. Robbin C. Moran, DEB 0717056). We thank two anonymous reviewers for their valuable comments, Patricia Eckel for revising the Latin diagnosis, and Mr. Haruto Fukuda for providing the line art illustration as well as John Mickel for allowing us to reproduce it here.

\section{Literature Cited}

Bishop, L. E. 1988. Ceradenia, a new genus of Grammitidaceae. American Fern Journal 78: 1-5. -1989. Zygophlebia, a new genus of the Grammitidaceae. American Fern Journal 79: 103118.

\& A. R. Smith. 1992. Revision of the fern genus Enterosora (Grammitidaceae) in the New World. Systematic Botany 17: 345-362.

Ching, R. C. 1940. On natural classification of the family "Polypodiaceae." Sunyatsenia 5: 201-268.

Copeland, E. B. 1947. Genera Filicum. Chronica Botanica, Waltham Mass.

- 1956 ["1955"]. Ctenopteris in America. Philippine Journal of Science 84: 381-473.

Evans, A. M. 1963. New chromosome observations in the Polypodiaceae and Grammitidaceae. Caryologia 16: 671-677.

Hasebe, M., P. G. Wolf, K. M. Pryer, K. Ueda, M. Ito, R. Sano, G. J. Gastony, J. Yokoyama, J. R. Manhart, N. Murakami, E. H. Crane, C. H. Haufler \& W. D. Hauk. 1995. Fern phylogeny based on $r b c L$ nucleotide sequences. American Fern Journal 85: 134-181.

Holttum, R. E. 1947. A revised classification of leptosporangiate ferns. Botanical Journal of the Linnean Society 53: 123-158.

Labiak, P. H. \& F. B. Matos. 2007. A new hybrid and two new combinations in neotropical grammitid ferns. Brittonia 59: 182-185.

, G. Rouhan \& M. Sundue. 2010. Phylogeny and taxonomy of Leucotrichum (Polypodiaceae), a new genus of grammitid ferns from the neotropics. Taxon 59: 911-921.

Lellinger, D. B. 1989. The ferns and fern-allies of Costa Rica, Panama and the Chocó (Part 1: Psilotaceae through Dicksoniaceae). Pteridologia 2A: $1-364$.
Mettenius, G. H. 1866. Filices; praesertim Indicae et Japonicae. Annales Museum Botanicum LugdunoBatavi 2: 149-313.

Morton, C. V. 1967. The genus Grammitis in Ecuador. Contributions from the United States National Herbarium 38: 85-120.

Murillo, M. T. \& A. R. Smith. 2003. Luisma, a new genus of Grammitidaceae (Pteridophyta) from Colombia. Novon 13: 313-317.

Nayar, B. K. \& S. Devi. 1965. Spore morphology of Indian ferns. IV. Grammitidaceae. Grana Palynologica 6: 121-127.

Newman, E. 1840. A history of British ferns and allied plants. J. Van Voorst, London.

Parris, B. S. 1990a. Grammitidaceae. Pp. 153-157. In: K. Kubitzki (ed.), The Families and Genera of Vascular Plants, Vol. 1. Springer-Verlag, Berlin, Heidelberg, New York.

Parris, B. S. 1990b. Noteworthy species of Grammitidaceae from South-East Asia. Hooker's Icones Plantarum 40: 1-129.

- 2003. Distribution of Grammitidaceae inside and outside Malesia. Telopea 10: 451-466.

.2007. Five new genera and three new species of Grammitidaceae (Filicales) and the re-establishment of Oreogrammitis. Gardens' Bulletin Singapore 58: 238-274.

- 2009. New genera of Malesian Grammitidaceae (Monilophyta). Blumea 54: 217-219.

Proctor, G. R. 1985. Ferns of Jamaica. British Museum (Natural History), London.

Ranker, T. A., A. R. Smith, B. S. Parris, J. M. O. Geiger, C. H. Haufler, S. C. K. Straub \& H. Schneider. 2004. Phylogeny and evolution of grammitid ferns (Grammitidaceae): a case of rampant morphological homoplasy. Taxon 53: 415-428.

Schneider, H., A. R. Smith, R. Cranfill, T. J. Hildebrand, C. H. Haufler \& T. A. Ranker. 2004. Unravelling the phylogeny of the polygrammoid ferns (Polypodiaceae and Grammitidaceae): exploring aspects of the diversification of epiphytic plants. Molecular Phylogenetics \& Evolution 31: 1041-1063.

Smith, A. R. 1992. A review of the fern genus Micropolypodium (Grammitidaceae). Novon 2: 419-425. . 1993, Terpsichore, a new genus of Grammitidaceae (Pteridophyta). Novon 3: 478-489.

\& R. C. Moran. 1992. Melpomene, a new genus of Grammitidaceae (Pteridophyta). Novon 2: 426-432.

- \& L. E. Bishop. 1991. Lellingeria, a new genus of Grammitidaceae. American Fern Journal 81: 76-88.

Sundue, M. A. 2010a. A monograph of Ascogrammitis, a new genus of grammitid ferns (Polypodiaceae). Brittonia.

- 2010b. A morphological cladistic analysis of Terpsichore (Polypodiaceae). Systematic Botany (in press).

, M. Islam \& T. A. Ranker. 2010. Systematics of grammitid ferns (Polypodiaceae): using morphology and plastid sequence data to resolve the circumscription of Melpomene and the polyphyletic genera Lellingeria and Terpsichore. Systematic Botany (in press). 
Tryon, R. M. \& A. F. Tryon. 1982. Ferns and allied plants with special reference to tropical America. Springer-Verlag, New York.

\& R. G. Stolze, 1993. Pteridophyta of Peru. Part V. 18. Aspleniaceae-21. Polypodiaceae. Fieldiana, Botany, New Series, 27: 1-176.
Wagner, F. S. 1985. Bilateral spores in new world grammitid ferns. American Fern Journal 75: 6-11.

Wilson, K. A. 1959. Sporangia of the fern genera allied to Polypodium and Vittaria. Contributions of the Gray Herbarium of Harvard University 185: 97-127. 